\title{
The effect of COVID-19-induced mortality salience on delay discounting: a replication
}

\section{Fatih Sonmez ${ }^{1}$ (]}

Received: 17 December 2020 / Revised: 10 October 2021 / Accepted: 26 October 2021 /

Published online: 5 November 2021

(c) The Author(s), under exclusive licence to Economic Science Association 2021

\begin{abstract}
This study is a conceptual replication of Kelley \& Schmeichel (PLOS ONE 10: e0144228, 2015), which found that thinking about death reduces delay discounting. Unlike the original study, the current study was conducted in an environment where there was a real and tangible mortality threat across the world, that is, COVID-19. Contrary to the findings of the original study, results of the current study revealed that thinking about death increases delay discounting, such that participants who were primed with death thoughts traded "£200 now" for "£342.35 three months later," whereas those in the control condition traded “牛200 now" for “生19.27 three months later". The current study also explored the moderating roles of goal orientation and self-esteem in the effect of mortality salience on delay discounting; however, it failed to provide evidence for the moderating roles of these variables.
\end{abstract}

Keywords Mortality salience $\cdot$ Delay discounting $\cdot$ Replication $\cdot$ COVID-19

JEL codes D91

\section{Introduction}

The vast majority of previous mortality salience studies were conducted in environments with little or no explicit real-life mortality threat; thus, participants of these studies confronted a hypothetical death. In contrast, the COVID-19 pandemic provides a natural setting for mortality salience research, offering a more valid ecological context for testing the effects of death thoughts. This situation calls for the

Fatih Sonmez

fsonmez.phd@gmail.com

1 Faculty of Economics and Administrative Sciences, Muş Alparslan University, Muş, Turkey 
replication of previous mortality salience studies. This study aims to replicate Kelley and Schmeichel's (2015) research, which tested the effect of mortality salience on delay discounting.

\section{Current study}

Like the original study, the current study is a one-factor between-subjects experiment (mortality salience: yes vs. no), where delay discounting was measured as the dependent variable.

\subsection{Participants}

Participants were students at Sakarya University Business Faculty who were at least 18 years old and had enrolled in several business courses. They were invited to participate in the study through an invitation letter posted on their student information systems. In the invitation letter, they were told that the study would investigate college students' general attitudes, and they were provided with the link to the survey on Qualtrics ${ }^{\mathrm{TM}}$. Participation was voluntary and was not compensated with either monetary reward or course credits. Ethics approval was obtained from the Social and Humanities Ethics Committee of Sakarya University. At the end of the experiment, participants were offered free psychological support from a prearranged psychologist, if they needed it; however, none of the subjects expressed or showed any interest in this offer.

\subsection{Materials}

\subsubsection{Mortality salience manipulation}

Thoughts of death were manipulated via a text about COVID-19 and an open-ended question from the classic mortality salience manipulation (Rosenblatt et al., 1989). Participants in the mortality salience condition first read a short text about the deadliness of COVID-19, the vulnerability of people to the virus, and the ubiquity of reminders of death during the pandemic. The text ended with a closed-ended question that inquired whether participants had been contemplating death or mortality in that context. This question was intended to prepare participants for the subsequent open-ended question that asked about the feelings and thoughts evoked by their contemplation of death. The introductory text, the closed-ended question, and the openended question read as follows:

The coronavirus (COVID-19) pandemic, which to date has caused hundreds of thousands of people to die, continues to kill tens of thousands of people every day. As creatures vulnerable to this unexpected threat, we follow the statis- 
tics shared by officials every day, constantly encounter content that reminds us of death and mortality in social media and other media outlets, and possibly know of deaths within our social circle. Do you ever find yourself contemplating death nowadays?

1-Never, 2-Seldom, 3-Sometimes, 4-Often, 5-Always

How exactly do you feel when you remember that you will die one day, and what do you think? Please write down, as specifically as you can, the thoughts and emotions that thinking about your own death arouses in you.

Participants in the control condition underwent a parallel process but received prompts concerning a neutral topic on the world film industry and film awards. The wording of the text and the questions was comparable to that of the text and the questions given in the mortality salience condition. The introductory text, the closed-ended question, and the open-ended question read as follows:

The world film industry, which to date has presented hundreds of thousands of movies and series to audiences, continues to produce tens of thousands of new movies and series every year. As consumers who have to choose among the alternatives, we follow the content shared by the producers, conduct research about movies and series on social media and other media outlets, or we get movie or series recommendations from our social circle. Do you ever research the awards that the movies or series you'll watch have received?

1-Never, 2-Seldom, 3-Sometimes, 4-Often, 5-Always

When you find out that a movie or a series has received a significant award, what do you think about that movie or series? Please write down, as specifically as you can, the feelings and thoughts that noticing that the film has received an award arouses in you.

\subsubsection{Delay discounting}

In the original study, participants made a series of non-incentivized, hypothetical choices between receiving $\$ 50$ now or receiving other dollar amounts three months later, starting with $\$ 50$ and increasing by $\$ 5$ on each offer up to $\$ 100$. The value at which participants switched from the immediate reward $(\$ 50)$ to the three-monthslater choice was coded as the indifference point. We used the same task in the current study, but we changed the currency to the Turkish Lira (£). The choices were not incentivized, as in the original study. To determine a baseline amount for the current study, we compared the costs of living in College Station, the city where the original study was held, to the costs of living in Istanbul, using the Numbeo database. Our study was conducted in Sakarya, a city that is very close to Istanbul and offers the same living standards. However, since the information in Numbeo concerning Sakarya was not accurate, probably due to lack of data, we preferred Istanbul, which had more realistic data on the costs of living. After controlling for the inflation in the U.S. from 2015 (the original study's year of submission) to 2020, we found that $\$ 50$ in 2015 in College Station is comparable in purchasing power to 
approximately $€ 200$ in Istanbul in 2020 . Participants in the current study made nonincentivized choices between receiving $£ 200$ now or receiving other amounts three months later, starting with $€ 200$ and increasing by $€ 20$ on each offer up to $€ 400$. The point where participants switched from the immediate reward to the three-monthslater choice was coded as the indifference point, as in the original study.

\subsubsection{Attention check}

Since limited language proficiency could introduce noise in the data, we employed a seven-point question both to identify non-native participants and check for respondent attentiveness. Participants were asked to tick " 6 " if they were international students, but not to make a choice otherwise. Those who ticked " 6 " were identified as international students, and those who ticked any other option were identified as inattentive.

\subsubsection{Manipulation check}

We used a word fragment completion task as a manipulation check to assess the accessibility of death thoughts (Greenberg et al., 1994). Participants were presented with eight fragmented words, each of which was missing two letters. Half of the words could be completed only as neutral words, while the other half could be completed either as neutral or death-related words. For example, "Ö_Ü_" could be completed as ölüm (death) or as several neutral words, such as ödül (prize), öbür (other) or öküz (ox). Participants were asked to fill in the missing letters to form the first word that came to their minds and complete the task as quickly as possible. The number of words completed as death-related terms was regarded as indicative of the accessibility of death thoughts and used for testing the manipulation's success.

\subsection{Procedure}

Data were collected online via Qualtrics ${ }^{\mathrm{TM}}$ between June 18 and July 4, 2020, within a battery of measures for unrelated studies. Upon clicking the survey link in the invitation letter, participants first viewed the informed consent, and those who agreed with the terms proceeded to the survey. In the survey, participants first indicated their age and gender, and in the following two pages, they responded to Likert-type measures of value orientation, self-esteem, and attachment style for the purpose of unrelated studies. They were then randomly assigned to either the mortality salience or the control condition and presented with respective experimental texts and questions. Then, as in the original study, they were presented with the intertemporal choice measure immediately after the experimental questions. Choices were listed on the same page, and participants indicated their preferences for each choice in order. At this point, the replication procedure was completed, and participants proceeded to the next pages to respond to several other measures for unrelated studies as well as the attention check question. Finally, 
participants engaged in a word completion task that served as the manipulation check, and then they were debriefed and thanked.

\subsection{Sample size and statistical power}

Data collection was conducted in an environment where lockdowns were imposed occasionally and face-to-face education was suspended; hence, it was uncertain whether we would achieve a pre-specified sample size. For this reason, we tried to reach as many participants as possible instead of specifying an a priori sample size. We achieved a larger sample size than that in the original study (i.e., 118 participants; $78.3 \%$ females; $M_{\text {age }}=21.19, \mathrm{SD}=2.88$ ) and fairly adequate power to replicate it. In total, 231 participants took the survey, 39 of whom dropped out before the intertemporal choice measure, while six skipped or did not properly respond to it (i.e., multiple switching). Eight participants failed the attention check, and 11 were international students. These cases were excluded from the analysis, leaving a final sample of $167\left(58.1 \%\right.$ females; $\left.M_{\text {age }}=22.89, \mathrm{SD}=4.47\right)$, which is sensitive to detect an effect size of $d=0.39$ with $80 \%$ power and affords 93\% power to detect an effect equal in size to the one reported in the original paper $(d=0.49)$ in a one-sided independent samples $t$ test with an alpha level of 0.05 (Faul et al., 2007).

\subsection{Results}

\subsubsection{Manipulation check}

For each participant, we recorded the number of words that were completed as death-related terms. We then conducted a $t$ test to compare the experimental groups in terms of death-related word count. Confirming the success of the manipulation, participants in the mortality salience condition $(M=1.03, \mathrm{SD}=0.96)$ produced significantly more death-related words than the control participants $(M=0.66$, $\mathrm{SD}=0.74), t(157)=2.71, p=0.007 ; d=0.43$.

\subsubsection{Hypothesis test}

To test the effect of mortality salience on delay discounting, we conducted an independent samples $t$ test. Contrary to the findings of the original study, our results showed that participants primed with death thoughts have a significantly higher delay discounting rate $(M=0.71, \mathrm{SD}=0.36)$ than those who engaged in a deathneutral topic $(M=0.60, \mathrm{SD}=0.37), t(165)=2.03, p=0.044, d=0.32$. In monetary terms, participants in the mortality salience condition traded "£200 now" for “£342.35 three months later", whereas participants in the control condition traded “£200 now" for “£319.27 three months later". We also conducted the same analysis without excluding participants to check whether exclusion had an impact on the 


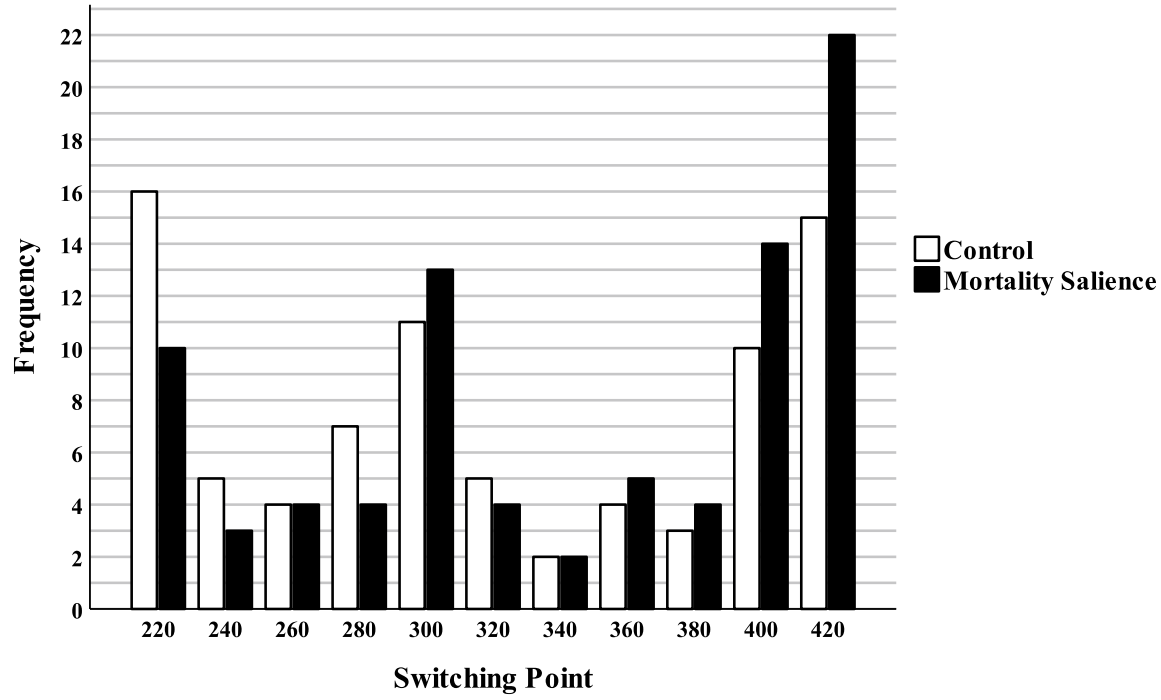

Fig. 1 Distribution of switching points across the experimental groups

results. This analysis revealed a significant effect in the same direction $(p=0.01)$, attesting the robustness of the results.

We also examined the distribution of the switching points across experimental groups. As can be seen in Fig. 1, although the distributions are generally similar across conditions, there are discernable differences in the frequency of lower and higher extreme values. Compared to participants in the control condition, participants in the mortality salience condition more frequently exhibited extreme discounting and less frequently switched at the lower extremes, showing that the overall difference between the two groups is largely accounted for by these differences in lower and higher extreme values.

\subsubsection{Exploratory analyses}

We explored the potential moderating roles of two relevant factors-goal/value orientation (intrinsic vs. extrinsic) and self-esteem-in the effect of mortality salience on delay discounting. We conducted two-step hierarchical regressions for each factor. In each model, we first entered the treatment variable (mortality salience; dummy coded) and the potential moderator (goal orientation or self-esteem; mean-centered), and then in the second step, we introduced their interaction terms into the models. However, neither the main effects of goal orientation $(p=0.3)$ and self-esteem $(p=0.33)$ nor their interactions with mortality salience $(p=0.38$ and $p=0.57$, respectively) were statistically significant. The main effect of mortality salience was significant in the model involving goal orientation $(p=0.035)$, and it was slightly above the conventional level of statistical significance in the model involving self-esteem $(p=0.054)$. 


\section{Discussion}

In the current replication study, we found a statistically significant effect in the opposite direction than in the original study. However, our study differs from the original study in a number of aspects, which could explain the differences in results. First, the original study was held in a classroom, whereas the current study was conducted online due to lockdowns and school closures. Second, the original study employed two open-ended questions from the classic mortality salience manipulation to induce death thoughts, whereas the current study employed COVID-19-related content and one open-ended question from the classic mortality salience manipulation. As for the control condition, the original study employed two open-ended questions on dental pain, whereas the current study employed a neutral topic on film awards since the dental pain scenario could effortlessly trigger concepts such as doctor, hospital, health, etc., which could potentially remind participants of the current pandemic situation and might jeopardize the manipulation. These deviations from the original study may account for the differences in results.

Another possible explanation for the divergent results can be based on the same reasoning that the authors used to explain their findings in the original study. Drawing upon construal level theory (Trope \& Liberman, 2010), the authors reasoned that personal death is seen as a hypothetical future event that promotes abstract thinking, which consequently reduces impulsivity and delay discounting. However, the current study was conducted in a substantially different ecology where a severe global virus outbreak poses a serious threat to human life. As such, participants were reminded of a death with a higher likelihood since the threat is tangible and therefore closer, both physically and psychologically. From a construal level theory perspective, reflecting on death in such a situation can induce more concrete cognitions, which can alter people's reactions (Cozzolino et al., 2004; Soderberg et al., 2015). For example, thinking about death may have led participants to focus on the present and depreciate the value of the future due to high uncertainty regarding the future amid pandemic times. Future work can test this hypothesis by manipulating thoughts of death on the basis of psychological distance. Also, the dental pain scenario employed in the original study may have been perceived as closer than death and led to a higher delay discounting rate. Thus, replacing dental pain with a neutral scenario may have reversed the pattern. Future research should take this possibility into consideration.

Differences in results may also be because participants' responses have been influenced not by thoughts of death but by the economic uncertainty caused by the COVID-19 pandemic (Altig et al., 2020; Yue et al., 2020). Reminding participants of the pandemic and asking them to make financial decisions may have caused them to make their decisions under the influence of the economic uncertainty inherent to the current pandemic. More in-depth research is needed to understand the effect of mortality salience on delay discounting.

Funding This research received no specific grant from any funding agency in the public, commercial, or not-for-profit sectors. 
Availability of data and material Data is available at https://osf.io/azsjb/.

Code availability An SPSS Syntax to reproduce the results is available at https://osf.io/vqwru/.

\section{Declarations}

Conflict of interest The author declare that there is no conflict of interest.

\section{References}

Altig, D., Baker, S., Barrero, J. M., Bloom, N., Bunn, P., Chen, S., \& Thwaites, G. (2020). Economic uncertainty before and during the COVID-19 pandemic. Journal of Public Economics, 191, 104274.

Cozzolino, P. J., Staples, A. D., Meyers, L. S., \& Samboceti, J. (2004). Greed, death, and values: From terror management to transcendence management theory. Personality and Social Psychology Bulletin, 30(3), 278-292.

Faul, F., Erdfelder, E., Lang, A. G., \& Buchner, A. (2007). G* Power 3: A flexible statistical power analysis program for the social, behavioral, and biomedical sciences. Behavior Research Methods, 39(2), 175-191.

Greenberg, J., Pyszczynski, T., Solomon, S., Simon, L., \& Breus, M. (1994). Role of consciousness and accessibility of death-related thoughts in mortality salience effects. Journal of Personality and Social Psychology, 67(4), 627.

Kelley, N. J., \& Schmeichel, B. J. (2015). Thinking about death reduces delay discounting. PLoS ONE, 10(12), e0144228. https://doi.org/10.1371/journal.pone.0144228

Rosenblatt, A., Greenberg, J., Solomon, S., Pyszczynski, T., \& Lyon, D. (1989). Evidence for terror management theory: I. The effects of mortality salience on reactions to those who violate or uphold cultural values. Journal of Personality and Social Psychology, 57(4), 681-690.

Soderberg, C. K., Callahan, S. P., Kochersberger, A. O., Amit, E., \& Ledgerwood, A. (2015). The effects of psychological distance on abstraction: Two meta-analyses. Psychological Bulletin, 141(3), 525-548.

Trope, Y., \& Liberman, N. (2010). Construal-level theory of psychological distance. Psychological Review, 117(2), 440-463.

Yue, P., Korkmaz, A. G., \& Zhou, H. (2020). Household financial decision making amidst the COVID-19 pandemic. Emerging Markets Finance and Trade, 56(10), 2363-2377.

Publisher's Note Springer Nature remains neutral with regard to jurisdictional claims in published maps and institutional affiliations. 